\title{
TÉLÉ-VISIONS DU CORPS-SPORTIF
}

\author{
Jean-Pierre Esquenazi ${ }^{1}$ et Éric Pedon ${ }^{2}$
}

La prise en compte du regain d'intérêt porté au corps sportif dans notre société moderne fait apparaître clairement l'émergence du sportif de haut niveau comme nouvel acteur médiatique d'importance. L'exploitation, la promotion et la consécration du corps, entretenues par les pratiques médiatiques (télévision, presse, publicité), ont institué le corps du sportif, comme un corps "spectacularisé", à partir duquel peuvent s'engendrer les activités passionnelles. La réception de la mise en spectacle du corps-sportif, qu'induisent les mises en scènes médiatiques, et en particulier télévisuelles, doit dès lors être abordée comme un des faits essentiels qui permettent de comprendre le phénomène de médiatisation des passions sportives.

Nous essaierons d'abord de comprendre quelle transformation sémiotique subit le corps-sportif quand il devient corps-sportif-télévisuel. Nous saisirons alors le corps comme un signe parmi d'autres, et nous bornerons à appréhender quelles significations il prend dans le contexte du stade d'une part, de la retransmission télévisée d'autre part. Ceci nous conduira à analyser quel téléspectateur sportif construit la télévision: nous parlerons d'un "sur-spectateur", résultat

1 Professeur à l'Université de Metz, membre du Centre de Recherches et d'Études sur les Médias.

2 Maître de Conférences à l'Université de Metz, membre du Centre de Recherches et d'Études sur les Médias. 
des prouesses techniques du média. Enfin nous verrons quels rôles médiatiques peut jouer le corps sportif mis en scène à la télévision, en nous appuyant sur quelques exemples publicitaires.

\section{Le paradoxe du sport}

Le sport est fondé sur l'idée d'un exercice imposé au corps. Cet exercice est défini comme un jeu: c'est-à-dire qu'il commence par définir un espace-temps clos, où l'exercice va prendre place. Cet espace est isolé du reste de l'espace social: pendant la durée de l'exercice, les gestes des acteurs sont supposés ne pas avoir pas d'autres conséquences que dans son enceinte. Par ailleurs l'exercice est fondé sur un ensemble de règles extrêmement précises, et, finalement, totalement, arbitraires. Comme tous les jeux, le sport a besoin de définitions rigoureuses, afin de donner à l'espace clos proposé aux concurrents sa spécification. L'arbitraire des règles permet d'abord d'autonomiser l'espace du jeu, de rendre distinctes ses frontières, tout en maintenant le présupposé de leur imperméabilité. Et leur précision, le fait qu'elles n'ont pas d'autres justifications qu'elles-mêmes, contribuent à suspendre les règles sociales usuelles pendant la durée du jeu. L'espace du jeu est donc une forme anthropologique d'espace complémentaire de l'espace social: on y procède à une sorte d'inversion rituelle de ce qui caractérise ce dernier: le jeu est "irréel", en ce premier sens qu'il suspend la "réalité" telle qu'elle est habituellement vécue. Mais il fait plus en isolant un ensemble d'actions du reste des actions habituelles, plongé dans le continuum de la vie sociale qui, elle, n'admet aucune coupure autre que symbolique. Comme l'écrit Eugen Fink, «le jeu nous libère de la liberté, mais d'une façon "irréelle"» 1 . Ce dont nous sommes libérés en tant que joueurs, c'est du choix que nous pouvons avoir dans la vie sociale: les règles du jeu déterminent notre action. Mais nous savons que cela ne durera que le temps du jeu, temps suspendu et "irréel".

Le sujet qui joue peut, alors, être autre que lui-même, il endosse le masque du joueur, et n'être, pour le temps du jeu, que ce masque. Ce dernier 'n'est pas quelque chose avec quoi on joue, mais quelque chose en quoi on joue"2. Le joueur peut ainsi transformer son identité,

1 E. FINK, Le jeu comme symbole du monde, Paris, Éd. de Minuit, 1966 (60), p. 229.

2 lbid., p. 159. 
apparaître sous une forme qu'on ne lui connaît pas habituellement: on connaît ces joueurs de football réputés être des hommes doux, qui deviennent des "terreurs" sur le terrain. Certains jeux sont même fondés sur le changement ou la construction d'identités nouvelles, comme les jeux de rôles.

Les sports sont des jeux, mais ce sont des jeux qui possèdent une caractéristique remarquable: le masque du joueur est son propre corps. D'où le paradoxe du sport: espace d'irréalité parce qu'il est un jeu, il dépend essentiellement de ce qui fait de nous des êtres matériels, des êtres dans le monde. Le corps du sportif est le masque dont il se couvre pour jouer: «Le monde du jeu est une apparence irréelle qui "saisit" les acteurs et les couvre sans cependant les cacher»'. Chaque geste du sportif exhibe son corps, en tant que ce dernier est l'apparence qu'il possède dans le cours du jeu. Ainsi il faudrait supposer à tout sportif deux corps, ou deux apparences corporelles: son corpssportif, qui est son masque sur le terrain de jeu; et son corps socialisé, qui diffère peu de celui que chacun de nous possède, celui qui apparaît par exemple dans les interviews d'avant ou d'après-match. Remarquons que le sport prend soin de donner un nouveau nom à chaque corps-sportif: c'est le dossard, ou le numéro qu'il porte qui constitue ce nom lié à l'événement sportif.

A chaque fois que le corps-sportif interfère avec le corps socialisé, se produit une émotion ou trouble particuliers: on se rappelle des poings noirs levés des sprinters américains sur le podium olympique de 1968; aujourd'hui, ce sont plutôt les larmes de Sampras, après son match contre Courrier, qui émeuvent. Quoique ces "deux" corps se produisent dans un seul corps, ils doivent être distingués, et le sont toujours par le spectateur qui juge selon les règles du jeu sportif. On pourra dire qu'un joueur réputé "méchant" possède un "cœur d'or", une "famille adorable".

Le sport a donc cette particularité de faire se rejoindre une extrême "irréalité", celle du jeu, et une extrême "réalité", celle du corps: car le corps-sportif est toujours supposé être un corps "vrai", "naturel". L'entraînement ne fait que démultiplier des facultés innées: il contraint le corps à faire ce dont il est capable. Et le seul fait qu'on ne puisse pas pardonner à un sportif, c'est d'avoir tricher avec son corps-sportif, de s'être dopé.

1 Ibid., p. 110. 
Irréalité du jeu et réalité du corps demeurent intimement liées, et le charme du sport réside dans leur alliance extraordinaire. D'où vient que le sportif peut devenir un héros, que certaines images demeurent gravées dans les mémoires comme des mythes "vrais": la course de Owens aux J.O. de 1936, ou le bras bandé de Beckenbauer en 1970 lors de la finale de la Coupe du monde de football. Le corps qui se produit sur le terrain de jeu est toujours traité comme un signe: il est le signe que le sportif laisse apercevoir de lui-même sur le terrain. Parmi toutes les identités qu'un individu peut prendre, parmi toutes les manifestations de lui-même qu'il peut produire, celle d'un corpssportif est originale parce qu'elle est muette, si on fait abstraction des indications données aux partenaires, ou aux échanges avec l'arbitre. Seul le corps "parle", exprime la personne. Ce fait explique, parmi d'autres, qu'une équipe puisse être interprétée comme un seul corpssportif, lorsque cette équipe se révèle particulièrement dans son caractère de collectivité.

\section{Significations du corps-sportif}

Le corps-sportif est, pour le dire brièvement, la forme significative que revêt le sujet humain quand on l'examine dans l'optique d'une sémiotique du sport. Examinons le rôle que joue ce signe particulier à l'intérieur de cette sémiotique.

Le corps-sportif s'affronte directement aux éléments mis à sa disposition par les règles du sport concerné: les dimensions d'un terrain, ses obstacles, ses adversaires, etc. Son comportement est dicté par la loi arbitraire qui a fait du sport pratiqué ce qu'il est. Si on examine les rapports entre le sport et le corps-sportif, on peut donc assimiler le second à l'indice du premier'. On sait que le philosophe américain Peirce appelle justement indice un signe qui se rapporte à son objet . Mais si on rapporte le corps-sportif à l'individu exprimé, il en est plutôt l'icone: l'individu se présente sur le terrain de sport comme une certaine "qualité corporelle" de lui-même.

On pourrait objecter ici que, pour un connaisseur, le corps-sportif peut être aussi un symbole sportif. On sait que, pour Peirce, un

1 Pour être tout à fait rigoureux, on devrait dire que le corps-de-sportif est un indice d'une réplique (token) du sport en question: celle qui se manifeste par les particularités du terrain, de la partie en cours, de l'adversaire, etc. 
symbole est un signe qui se rapporte à son objet en fonction d'une loi ou d'une règle: le corps-sportif pourrait exprimer une sorte de perfection du jeu, une haute application de la règle. Le paradoxe du sport -irréalité pure et réalité incontestable mêlées- devient tangible, miraculeusement concret. Mais on voit alors que le signe ne peut pas être seulement le corps-sportif, mais celui-ci en tant qu'il est aussi un individu réfléchissant. Nous nous restreindrons donc, pour l'instant, aux qualités fonctionnelles du corps-sportif.

Cependant, qu'on lui donne comme objet le sport ou l'individu qu'il est par ailleurs, le corps-sportif est d'abord un signe-objet, un signe qui est une chose: ce que Peirce appelle un sinsigne 1 . Un sport est un jeu où les signes retenus le sont en fonction de leurs qualités matérielles: d'une part, un terrain dont les propriétés sont spécifiques, éventuellement un ballon, etc.; d'autre part des individus dont les capacités physiques et la conscience des règles représentent les principales conditions nécessaires à leur participation.

Participant à la représentation sportive, le corps-sportif est fondamentalement un objet réel qui s'affronte à la forme d'espacetemps sous laquelle le sport apparaît pour lui (un sinsigne indiciaire dicent, dirait Peirce). Pour être plus précis, dans la grammaire propre au sport, le signe corps-sportif demande à être interprété en tant qu'il désigne la réalité d'un être physique, d'une "physicalité". Et quand il apporte un surcroît de signification, par exemple passionnelle, c'est en réinterprétant cette "physicalité" comme une aventure corporelle: la souffrance d'un coureur de fond, l'exploit que représente une course ou une détente particulière, la rapidité d'une action collective, sont le développement du face-à-face (du corps à corps) entre le corps-sportif et les éléments matériels du jeu.

\section{Le corps-sportif-télévisuel}

Le sport trouve à la télévision une terre d'accueil privilégié. Il est patent que l'association entre direct et sport permet à la télévision de réaliser des performances d'écoute remarquables. Aujourd'hui, la télévision majoritaire a su associer un type de discursivité à un

1 Le corps-de-sportif est en lui-même un sinsigne: un signe qui est une chose. Rapporté au sport il est donc un sinsigne indiciaire, et rapporté à lui-même comme individu, un sinsigne iconique. 
ensemble de technologies dont le direct constitue le fleuron le plus éminent ${ }^{1}$. Si on se rappelle que le direct désigne la capacité de la télévision à maintenir une actualité virtuelle dans son immédiateté brute, à présenter une durée pure, sans passé ni avenir mais effectuant une présence absolue (qui feint d'être absolue), on comprend pourquoi le sport, ou plutôt l'événement sportif passe si aisément à la télévision: un tel événement constitue une partie, qui se déroule dans un espace-temps clos, parfaitement isolé du milieu social. Une telle partie possède, elle aussi, une durée pure. En cela, elle demeure prise dans l'irréalité propre au jeu. Mais elle lui ajoute la réalité des corpssportifs qui participent à l'événement. La télévision trouve ici ce qui lui manque la plupart du temps, un tangibilité, une évidence, qui ne peuvent être discutées. L'événement sportif s'insère dans un calendrier, dans une chronologie d'événements (coupes d'Europe, tournoi des cinq nations, championnat du monde, etc.) qui lui garantit une cohérence publique. Ainsi, l'événement sportif est à la fois parfaitement prévisible en tant qu'événement (ce qui manque cruellement à l'actualité...), et absolument imprévisible dans son déroulement (la glorieuse incertitude du sport...). La télévision parvient donc facilement à programmer ses diffusions, et profite de la valeur ajoutée de la retransmission sportive: l'indicialité du sport, la "physicalité" du corps sportif; en bref, tout ce qui assure au téléspectateur l'authenticité du spectacle proposé.

A ce point qui est capital, il faut en adjoindre un autre, caractéristique de la télévision contemporaine: l'irréalité du jeu sportif, qui extrait du social l'événement sportif, qui construit ce dernier comme un fait isolé, qui permet d'oublier que le sport, lui, est un fait social parmi d'autres, satisfait aussi la télévision; celle-ci ignore le social pour mieux lui substituer un social télévisuel, et elle n'aime rien moins que le rappel à l'intérieur de l'événement sportif de ses déterminations qu'elle se plaît à nommer "extra-sportives": voir le refus de l'émission Téléfoot, sur TF1, de traiter l'affaire OM-VA, avant de longues semaines, bien qu'il y ait aussi des raisons circonstancielles.

Ce goût de la télévision pour le sport justifie ses efforts pour faire du sport télévisé un genre d'émission privilégié: la télévision a su déployer des fastes de mise en scène, qui ont fait plus que donner une valeur ajoutée à la retransmission; la télévision a transformé le

1 Sur ce point nous nous permettons de renvoyer à J.-P. EsQueNAZI, Le pouvoir d'un média: TF1 et son discours, Paris, L'Harmattan, 1996. 
spectacle sportif, passionné les débats autour du sport, et accru fortement l'impact des corps sportifs.

Pour caractériser la représentation télévisée d'émissions sportives, le plus simple est peut-être de comparer le spectateur au téléspectateur. Rappelons d'abord le fait évident que, présent sur le stade (ou ce qui en fait office), le spectateur devient physiquement participant au spectacle sportif, tandis que le téléspectateur se contente d'une présence virtuelle. Et posons les questions concernant la perception du spectateur et celle du téléspectateur: que voit-on et qu'entend-t-on sur le stade, que voit-on et qu'entend-t-on à la télévision? Une première réponse, tracée à traits larges, pourrait être celleci:

- on entend, d'une part, la clameur du public auquel on appartient, et, d'autre part, le commentateur; il est vrai que l'ambiance du stade nous est également rendue, mais le commentateur occupe la place principale sur la bande sonore.

- on a, d'une part, une vision d'ensemble du terrain de jeu, et, d'autre part, une vision rapprochée de parties de ce terrain et des participants les plus concernés par l'action en cours.

L'apport du commentateur consiste principalement à nommer les joueurs selon leur identité civile; le spectateur se contente souvent des identités sportives, c'est-à-dire des numéros de maillot ou de dossard. Il privilégie le global et ne perd jamais de vue que le corps-sportif est un élément d'une structure générale. La vision rapprochée que permet la télévision met en valeur l'action individuelle de chacun des corpssportifs présents sur le terrain. Il semble que la multiplication des caméras n'a ni pour but ni pour effet de redonner une vision globale selon une fragmentation calculée des points de vue, mais de s'assurer qu'aucune action particulière n'échappera à la vigilance des caméras. C'est par exemple le point de vue, affirmé haut et fort, de la chaîne Canal +, dont on dit qu'elle est à la "pointe" de la retransmission sportive. Seul parmi les sports les plus retransmis, le tennis, semble-til, échappe à cette parcellisation. Mais les arrêt nombreux dans le cours du jeu permettent de revenir sur chacun des joueurs; de plus, le tennis possède une forme propre de narrativité à rebondissements qui se substitue à la diversités des actions des sports collectifs. Ainsi, nous pouvons dire que la vision télévisée favorise le sportif au détriment du sport lui-même.

La télévision assure au corps-sportif une double visibilité: 
- Elle en détaille les performances techniques. Nous ne pouvons plus ignorer le passement de jambes du footballeur, les différences entre la foulée du sauteur de haies et celle du sprinter, le contre du basketteur, etc. Le corps-sportif télévisuel met en avant la technicité du corps-sportif usuel: la source de l'interprétation du second par le premier se trouve dans la qualité de la réponse aux conditions concrètes du jeu. Le ralenti est ici un outil essentiel, permettant de détailler la sûreté du geste, sa pureté ou sa justesse.

- Elle le nomme avec une précision grandissante (la connaissance d'une biographie détaillée des sportifs paraît une condition nécessaire pour être admis au rôle de commentateur). Cette nomination ne renvoie, finalement, ni au nom social porté par le sportif, ni à l'identité que lui donne l'événement. Le "Maradona" que décrivent les commentateurs, ne se confond pas avec le joueur qui porte le numéro 10 , ni avec l'homme "Maradona" qui a su par le football atteindre un statut social qui lui était a priori interdit. Ce nom télévisuel condense un ensemble d'exploits diffusés dans le monde entier, une série de résultats (le "palmarès"), une carrière (le joueur est passé de tel club à tel autre, etc.). On pourrait dire que la représentation télévisée du sport produit une forme particulière d'identité attribuée aux sportifs ${ }^{1}$.

Si on admet notre hypothèse, on peut comprendre les transformations sémiotiques que la transmission télévisée fait subir au corpssportif, comme un processus d'individualisation du sportif lui-même: le corps-sportif-télévisuel bénéficierait d'un traitement permettant de construire autour de ce corps une personnalité sportive, aux traits personnalisés bien marqués.

Il faut d'abord constater que ces transformations du corps-sportif en corps-sportif-télévisuel prennent la forme de réinterprétations: le second ne prend sens dans la mesure où la connaissance du premier est présupposée (en termes peirciens, on dira que se forme un interprétant second, dont le signe de référence est l'interprétant du signe premier "corps-sportif"). Le téléspectateur doit connaître les règles qui gouvernent la pratique sportive, et son fonctionnement sémiotique; le sens propre produit par la télévision s'ajoute à un sens général premier, qui constitue l'interprétation admise du sport.

1 Bien sûr, la télévision n'est pas seule responsable de la production de cette identité. Les journaux spécialisés y contribuent notablement. Mais la télévision est, croyonsnous, responsable de leur diffusion. 
Nous avons signalé deux de ces réinterprétations: la première saisit dans l'indicialité du corps-sportif une qualité particulière du corps en action, propre à ce corps-là (le "toucher" d'Edberg, la "puissance" de Koeman, la "hargne" de Rives). Ces qualités font l'ordinaire des propos des commentateurs, qui fondent sur elles la plupart de leur propos. Il s'agit d'une opération consistant à retirer d'un type physique un élément qualitatif propre à décrire le comportement général du sportif, son allure propre.

Nous avons vu que le corps sportif se présente comme l'icone de l'individu pratiquant le sport. Depuis que la retransmission télévisée a transformé les enjeux du sport, la présentation de soi s'est largement métamorphosée. Les maillots ou tenues sportives sont l'objet d'un soin extrême. Jeunes femmes vêtues de collants moulants et étincelants, tennismen qui abandonnent l'uniforme blanc pour diverses modes de bariolages, sont filmées souvent de très près, lors des moments de préparation ou d'arrêt du jeu. Cette attention nouvelle à l'iconicité se rapporte moins à l'identité sociale du sportif mais à son identité sportive, construite par les médias eux-mêmes à partir de l'histoire du sportif.

La troisième s'approprie une histoire sportive pour décliner l'attitude du joueur en termes d'"expérience" ou de "naïveté" (la "ruse" de Fernandez, le "calme" de Borg). La connaissance de l'identité sportive du joueur autorise l'érection d'une loi d'interprétation de son évaluation du jeu, de son aptitude à comprendre le déroulement de l'événement. Cette définition d'une identité ouvre ensuite aux sportifs d'autres espaces que ceux du terrain; elle constitue le principe premier qui va leur permettre d'apparaître sur d'autres scènes, qu'elles soient publicitaire, ou médiatique.

\section{Le spectacle du corps-sportif}

Il est donc aisé de constater que la télévision se nourrit du sport tout en le construisant conformément à ses intérêts propres. Au demeurant, on sait combien l'audimat, les enjeux financiers et publicitaires et la logique économique des chaînes ont modifié le paysage du sport à la télévision, et comment celle-ci a imposé une hiérarchie entre les disciplines sportives en privilégiant celles qui répondent au mieux aux critères de visibilité, de spectacularité, de dramaturgie, de scénari- 
sation qu'exige l'écriture télévisuelle. Si l'influence de la télévision est déterminante dans la construction de la représentation des sports qu'elle propose au regard des téléspectateurs (tout média construit son objet de discours selon sa logique et ses fins), il nous semble utile de montrer également en quoi le corps-sportif-télévisé en tant que figure sémiotique complexe constitue un des enjeux centraux de la jouissance spectatorielle, et, ainsi, alimente un des aspects des passions sportives.

Si chez le téléspectateur, le plaisir d'assister à un exploit sportif, la fascination pour le mouvement parfait, le geste pur, du sportif de haut niveau, l'attrait pour la motricité exemplaire du champion professionnel, connaissent une forme particulière d'intensité, cela tient à une des formes induites par le processus d'individualisation, décrit précédemment, que la télévision, par ses modes de médiatisation du spectacle sportif, fait subir au corps-sportif. Dans les images des manifestations sportives, la télévision s'attache avec insistance, par le développement important de moyens techniques et humains ainsi que par le renouvellement permanent des dispositifs de regard, à augmenter le degré de visibilité du corps-sportif et à rendre compte, dans ses moindres détails, de son expressivité. L'ostension paraît être le dénominateur commun aux mises en scène télévisuelles. La mise en images (par la variation des points de vue et la sélection des plans) et l'écriture télévisuelle spécifique (par le montage, les ralentis, les retours en arrière) tendent non seulement à maîtriser l'espace et le temps de la représentation, mais visent aussi à abolir la distance entre le regard du spectateur et le corps-sportif. Elles proposent ostensiblement, au téléspectateur, une vision très proche du corps regardé, vision qui s'idéalise dans la simulation d'un "face-à-face" ou d'un "côte-à-côte", impossible dans la réalité, entre le spectateur et le sportif. La télévision, par son insistance, par sa vision rapprochée, par son savoir encyclopédique, ne se contente pas de restituer le corpssportif en action au téléspectateur: elle le désigne à son attention, elle en montre et remontre l'art, elle en célébre l'histoire singulière. Par là, elle démontre son pouvoir: elle redouble le "voici un corps-sportif", d'un "voici les moyens de la télévision". Du même coup, en affichant sa technologie, elle crée ostensiblement un corps-sportif-télévisuel en réunissant ses propres performances et celles du corps-sportif. 
Une des conséquences de l'ostension, réalisée par les moyens techniques de la télévision vis-à-vis du corps-sportif, concerne l'opération de transformation du spectacle sportif originel en un spectacle télégénique. Dans ce dernier, l'expressivité du corps-sportif y est présente sous toutes ses manifestations: le corps-action (efforts, gestes spécifiques), le corps-émotion (affects euphoriques/ dysphoriques, réactions, cris, tics, tensions), le corps-réflexion (concentration, pensées), que le téléspectateur peut voir, revoir, en gros plan, sous différents angles, au ralenti. La télé-vision est autrement supérieure à la vision d'un spectateur présent dans l'enceinte où se déroule la manifestation sportive: elle relève d'une ingénierie du regard, où la technologie ajoute ses images aux images du spectacle, où le regard technologique redouble le regard humain. Lors des derniers championnats du monde de natation en 1995, outre les habituelles caméras permettant de suivre en travelling, en plan général et en gros plan, le déplacement des nageurs, ou de voir sous l'eau les virages, une caméra mobile placée au fond du bassin proposait la vision par en-dessous des mouvements des nageurs. Le téléspectateur pouvait ainsi voir, durant un temps assez court, ce qui est impossible de voir pour un spectateur assis dans les gradins.

Cette performance technique de simulation d'une présence regardante, qui produit une vision amplifiée (l'invisible devient visible) propose finalement au téléspectateur une place virtuelle d'où il peut jouir de façon optimale du spectacle du corps: tout en étant éloigné, il peut voir le maximum, de près et de partout. On peut ainsi expliquer le succès du sport à la télévision comme divertissement, pour toutes les couches sociales et notamment au sein de la population masculine, par les possibilités de participation du téléspectateur au spectacle sportif, permises par les dispositifs du "mieux voir" et du "tout voir" qu'innovent à chaque événement sportif d'importance les chaînes de télévision. On peut également comprendre pourquoi le téléspectateur peut éprouver par procuration les sensations de la pratique sportive filmée. Le simulacre de la vitesse dans les sports mécaniques est sans doute la sensation la mieux traitée par la télévision. La caméra fixée sur les voitures de F1, véritable "œil greffé" faisant corps à la machine qui devient du même coup voyante, en adoptant un point de vue proche de celui du champion ou même impossible pour un témoin humain, procure une vision qui fait réellement sensation. Cette sensation devient paroxystique lorsque survient l'accident: les images, transmises pendant l'impact et la trajectoire devenue incontrôlable de 
la voiture, rendent compte réellement de la réalité physique perçue par le pilote, et le simulacre devient quasi-perception. On se souvient des images vues "de l'intérieur" lors du looping spectaculaire de R. Patrese au Grand Prix du Portugal en 1992, des images fortes de l'accident mortel de A. Senna vues depuis la voiture de M. Schumacher au Grand Prix d'Italie en 1994, de celles de la voiture de M. Hakkinen au Grand Prix d'Australie en 1995.

D'où l'idée que le spectacle du corps-sportif à la télévision donne finalement naissance à un "sur-spectateur" fabriqué par elle, se divertissant des exploits physiques de "sur-hommes", vibrant au rythme des corps-sportifs incarnés par les nouveaux héros idolâtrés dans l'arène médiatique mondiale. Entre corps-sportif et corps-spectatoriel, la passion s'installe à partir de l'interaction générée par les dispositifs télévisuels du regard, et qui selon trois ordres: le réel, l'irréel et le virtuel. A propos de cette interaction, on commence à prévoir une nouvelle révolution technologique, avec l'arrivée annonçée de la télévision interactive. Deux expériences méritent d'être soulignées. Le système Videoway, développé par la chaîne canadienne Videotron, permet au téléspectateur de devenir "télé-acteur": il peut composer grâce à sa télécommande son propre spectacle sportif, en zappant sur quatre canaux transmettant la même rencontre sportive ; chaque canal propose un point de vue particulier : neutre, technique ou partisan d'une des deux équipes. On a prévu aux États-Unis de permettre au téléspectateur de pouvoir, au cours d'un match de football, obtenir les fiches biographiques de chaque joueur, revoir les moments-clés à tout instant, au ralenti et sous un autre angle, et même participer aux discussions stratégiques du jeu. Avec la télévision interactive, le "surspectateur" aura la possibilité (toujours limitée) de jouir du spectacle sportif à double titre: celui de récepteur et celui d'un pseudo-réalisateur de programmes destinés exclusivement qu'à lui-même.

\section{Le corps du spectacle médiatique}

Dès lors, on remarque combien l'enjeu de la représentation s'est déplacé: ce n'est plus tant le sport et sa dimension compétitive et/ou ludique que le corps et ses exhibitions spectaculaires qui se trouvent privilégiés par la télévision. L'individualisation du corps-sportif, résultant des mises en scène et des mises en discours du spectacle sportif par les chaînes, accompagne évidemment l'évolution moderne 
du sport dans sa dimension sociale (amateurisme/professionnalisme, pratiquant anonyme/star mondiale), avec la montée en puissance des enjeux financiers. Comme nous l'avons indiqué plus haut, ce processus d'individualisation est lié au triple fonctionnement sémiotique du corps-sportif (indice, icône, symbole). Ce dernier renvoit davantage à l'image du sportif qu'à l'image du sport: il désigne plus une corporéité qu'une sportivité, et cette caractéristique lui permet d'être introduit dans des domaines extra-sportifs, mais où l'image du corps et ses valeurs comptent. Nous pensons en particulier à la publicité, mais aussi à la mode, au prêt-à-porter, et plus généralement aux styles de vie et de comportement fondés sur le culte du corps. La mise en spectacle du corps-sportif lui confère un statut complexe et un ensemble de valeurs, qu'actualisent l'hétérogénéité des usages et les multiples images que composent les scénarios et les scénographies issues de l'ensemble des pratiques sociales.

A la télévision, dans les programmes sportifs, le corps-sportif est ostensiblement désigné, affiché, décrit, analysé, commenté, interprété, selon les régimes du voir et du savoir, que, pour sa part, S. Haefliger désigne respectivement par les termes de rhétoriques du "rapport" et du "report"1. Dans l'espace social, la spectacularisation du corpssportif s'effectue selon plusieurs modes d'expression, qui relèvent de ce qu'on pourrait désigner comme étant une rhétorique de l'“import": l'exhibition, l'emprunt, l'imitation, la fictionnalisation, la dérivation. Mais, tant dans les rhétoriques du "rapport" et du "report" que dans celle de 1'“import", la qualification du corps-sportif reflète un ensemble de valeurs ajoutées qui dépasse la valeur purement sportive. De fait, nous considérons que le corps du spectacle médiatique, signifiant au-delà du spectacle sportif, syncrétise les trois pôles du signe corps-sportif: il est à la fois performant (indice), esthétique (icone) et normatif (symbole).

Le corps-sportif signifie un corps performant, résultant d'une maîtrise corporelle poussée aux limites humaines; la réussite sportive est liée aux qualités physiques et techniques, développées par un travail long et scientifique, et appliquées par l'individu sportif au cours de sa pratique, dans un espace-temps plus ou moins réglé.

${ }^{1} \mathrm{~S}$. HAEFLIGGER, "Une analyse sociographique de la rhétorique médiatique du sport", La quatrième mi-temps, Louvain-la Neuve, Observatoire du récit médiatique, 1995, p. 127-138. 
L'exploit individuel est l'indice que l'homme a maitrisé au mieux son corps, le milieu dans lequel il évolue, les objets qu'il utilise, l'espace et le temps. Par le biais de la télévision, le public a tendance à privilégier les gestes les plus spectaculaires, qui allient force, vitesse, adresse, technicité. Sa passion se réduit au geste exemplaire et rare, où tout s'harmonise en un instant mémorable. Cela est bien visible dans la rubrique Top Buts de l'émission Téléfoot sur la chaine française TF1, où les téléspectateurs élisent les plus beaux buts du championnat: les premières places sont régulièrement occupées par les buts inscrits sur une reprise de volée, ou mieux encore, par un retourné acrobatique.

Si le corps-sportif vise à travers la performance à repousser sans cesse les limites humaines, à nier les qualités ordinaires, la création publicitaire vient bouleverser l'ordre naturel en concevant un monde où le corps performant des vedettes est fictionnalisé à outrance dans l'excès. Deux exemples de spots publicitaires empruntés à la marque Nike sont significatifs de cette tendance. Le premier met en scène le célèbre basketteur américain Michael Jordan. Dans un hangar, au fond duquel se trouve un panier de basket, la vedette s'élance en dribble, puis grâce à une impulsion phénoménale, s'élève vers le panier pour effectuer un smash puissant. Le saut dure interminablement, le spectateur ayant ainsi le temps d'apprécier l'équilibre parfait du joueur. Le spot se termine par un gag: le joueur reste accroché au panier et regarde vers le sol; on s'aperçoit en fait qu'il est à plusieurs mètres du sol. Le message est clair: si M. Jordan, déjà doté de qualités exceptionnelles, a pu réaliser un exploit impossible dans la réalité, c'est grâce à ses chaussures ultra-performantes. La fiction invraisemblable de ce spot fonctionne sur les principes de l'assimilation et de la dérivation: chez Nike, la qualité de détente caractérise les chaussures et défie les lois physiques. On retrouve ce registre de l'invraisemblable dans le second spot publicitaire où s'affrontent André Agassi et Pete Sampras. L'échange démarre sous les yeux du commentateur qui n'est autre que John Mc Enroe. Rapidement, on s'aperçoit que la durée de l'échange n'est pas ordinaire: la nuit tombe, Mc Enroe sommeille, les spectateurs sont allongés dans les gradins, les saisons passent, l'herbe pousse sur le court. Le spot se termine sans que l'échange soit conclu. De nouveau, le message est évident: les chaussures Nike défient la loi du temps, elles endurent sans faiblir, à l'image des deux vedettes qui se renvoient coup pour coup. Dans ces produits publicitaires (d'autres marques sportives pourraient être également citées, telles que Puma ou Diadora), l'assimilation et la dérivation de la nature de la perfor- 
mance sont poussées à l'extrême: les valeurs de performance du corpssportif sont extrapolées vers l'objet auquel on ne reconnait plus une fonction ordinaire (chausser les pieds), et les vedettes ont désormais besoin d'objets supra-ordinaires pour défier les lois du sport, de l'espace et du temps et réaliser des performances sur-naturelles.

Le corps-sportif s'appréhende comme un objet esthétique, soumis au regard du public et des médias, tant au niveau de ses modes d'apparition qu'au niveau de sa forme plastique et de son expressivité gestuelle. Le port de vêtements collants, colorés, échancrés, légers, qui épousent les formes, mettent en valeur les lignes, affinent les silhouettes, dévoilent ou dénudent certaines parties, souligne le goût des apparences et le culte de la beauté chez les sportifs. L'originalité ou l'élégance du corps-sportif est affaire de musculation, de souplesse, d'allure, mais aussi de "look". Toute vedette se doit d'être attentive en permanence à son style vestimentaire, à sa parure, pour être reconnaissable immédiatement: André Agassi et ses tenues excentriques, Eric Cantona et son col de maillot relevé, les handballeurs français et leurs coupes de cheveux originales, les footballeurs de l'A.C Milan et leurs costumes à la sortie des vestiaires. Leur corps singulier n'est pas seulement un instrument sophistiqué et sculpté: il exige une mise en beauté particulière qui le distingue des autres concurrents.

Par le biais des caméras de télévision, le téléspectateur peut à certains moments apprécier les aspects virils ou féminins du corpssportif, vantés par les commentateurs: la force et la puissance du rugbyman Jonah Lomu ou de Mike Tyson, la beauté ténébreuse de Gabriela Sabatini, le charme gracieux de Katarina Witt. Grâce aux gros plans insistants et intéressés, il peut aussi jouir des formes corporelles plus intimes: le modelé des muscles (le torse sculptural et dénudé à la fin d'une course de L. Christie), la finesse des courbes (la cambrure impressionnante de M. Ottey), etc. De même, les ralentis permettent de mettre en valeur la beauté tonique du geste sportif, l'allure d'une déplacement, l'équilibre d'un saut, la grâce artistique d'une attitude, comme dans les épreuves de patinage artistique, dont l'audience grandissante auprès du public se traduit par une programmation en prime-time sur les chaînes françaises. Tout ceci souligne l'intérêt et la passion pour le sport conçu comme un art corporel total.

De plus, à notre époque, le corps-sportif se voit empreint d'une charge de séduction que la publicité, la mode et les médias s'approprient. David Ginola devient mannequin pour Cerruti, le temps d'un 
défilé de mode, ou pose nu pour un magazine féminin. Dans un spot publicitaire pour le parfum Rebel Rebel de Gemey, Fabrice Candeloro patine dans les rues de New-York à la poursuite d'une blonde au volant d'une décapotable rouge; la chemise ouverte sur son torse nu, il saute acrobatiquement au-dessus des motos de la police pour déposer un foulard rouge autour du cou de la belle, qui séduite par l'odeur du tissu, le poursuit à son tour et le rejoint pour une étreinte. Dès lors, le corps-sportif est perçu comme un canon de référence de l'esthétique corporelle, une icône moderne à laquelle s'attache une forte valeur de séduction, un emblème érotique. Son image médiatique met en jeu finalement une esthétique corporelle construite sur l'être et le paraître, sur la forme et l'expression, et dont l'iconicité repose sur la fonctionnalité et la beauté plastique qui se conjuguent dans l'harmonie et l'équilibre idéal des volumes, des lignes, des courbes, des angles. La médiatisation du corps-sportif peut alors être considérée alors comme la glorification d'un corps-design mis en scène.

Le corps-sportif possède une valeur symbolique, liée à sa fonction d'identité sociale et culturelle. Son image dans la société se fonde sur les facteurs archétypaux de réussite qui émergent avec l'histoire des sportifs professionnels. On sait que le sport, par sa dimension compétitive, peut être moteur d'ascension sociale, d'affirmation de soi, d'intégration sociale, de promotion culturelle, d'éducation. Par ailleurs, de nombreux auteurs ont mis en évidence l'importation des valeurs sportives dans le champ social, tant au niveau économique, qu'idéologique ou éthique. En particulier, comment la dynamique psychologique du corps-sportif et ses diverses manifestations (goût de la perfection, persévérance, ambition, contrôle de soi, responsabilité, maîtrise émotionnelle, adaptation, etc) constituent un modèle normatif dans notre société marquée par le culte de l'excellence.

Cette normalisation du corps-sportif comme signe d'une hygiène de vie, dont un des aspects est témoigné par l'essor des clubs de remise en forme, est bien visible dans la représentation médiatique des styles de vie, des comportements, des mentalités, empruntés à l'univers sportif, que véhiculent la télévision, la publicité, le cinéma, le prêt-à-porter. Les survêtements et les chaussures de sport se portent dans la rue, les hommes et les femmes pratiquent en loisir le jogging, la musculation, le vélo tout terrain, et sur les écrans, les corps sont en sueur. A la télévision, les reportages sur les sportifs professionnels 
célébrent leur réussite et leur intégration sociale, selon un modèle de scénario pratiquement identique. Prenons l'exemple du footballeur, le sportif le plus représenté à l'écran. On nous présente sa maison individuelle, son habitat coquet, sa femme (le plus souvent dévouée à la carrière de son mari) et ses enfants, sa voiture haut de gamme, c'est-àdire tous les stéréotypes d'une vie réussie et aisée. On le voit pratiquer des loisirs (lecture, télévision, promenades), puis partir à l'entraînement où l'attendent des supporters réclamant des autographes. Suivent les exercices physiques, les jeux de ballon, les conseils de l'entraineur, les instants de joie et de bonne humeur avec ses partenaires. En somme, nous avons la représentation d'une vie bien réglée, agréable, sans problèmes, dans laquelle le sportif est bien dans sa peau, bien dans sa famille et dans son club, bien avec les autres. On voit ainsi comment la médiatisation du corps-sportif dans les émissions de sport instaure une norme symbolique, une règle de vie individuelle et sociale qui, finalement, est transposable à tous, laissant aux journaux télévisés le traitement des écarts des sportifs par rapport à cette règle (le cas de Patrice Loko, joueur du PSG, pris d'un accès de folie, arrêté et interné).

L'image symbolique du corps-sportif, que proposent les médias et que la société a valorisée, apparaît bien comme un puissant vecteur de normalisation des corps anonymes, d'homogénéisation des styles de vie et de diffusion de modèles psycho-sociologiques.

\section{Pour conclure}

La télévision a profondément changé notre rapport au corpssportif; elle a d'abord transformé ses propriétés sémiotiques, c'est-àdire la manière dont il s'offre comme un signe. A la fois objet esthétique et machine ultra-performante, symbole de réussite et d'individualisation, le corps-sportif-télévisuel a supplanté le sport lui-même comme vedette des retransmissions. Mais elle a changé aussi notre regard sur ce corps: elle a constitué ce "sur-téléspectateur" évoqué plus haut, détenteur d'un oil électronique et d'un ordinateur encyclopédique. Bien sûr, point de vue et objet sont inséparables: la constitution du premier va de pair avec celle du second, l'ensemble produisant une nouvelle vision du sport. Vision qui privilégie l'individuel aux dépens du collectif, l'exploit aux dépens de l'assiduité et de la régularité: le corps y acquiert une visibilité remarquable et une valeur immédiatement monnayable. Mais ceci est une autre histoire... 